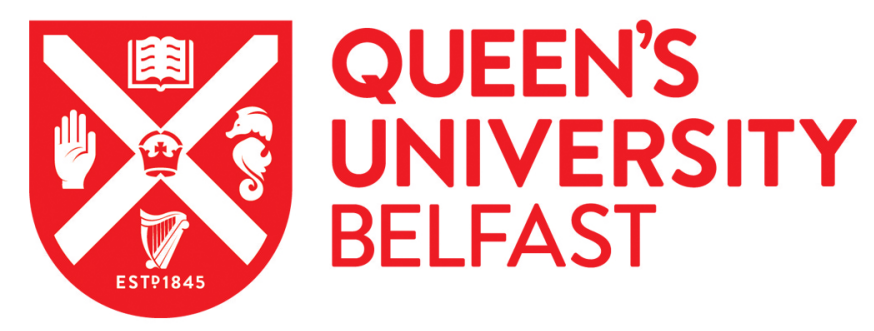

\title{
Discovery of interstellar anions in Cepheus and Auriga
}

Cordiner, M. A., Charnley, S. B., Buckle, J. V., Walsh, C., \& Millar, T. J. (2011). Discovery of interstellar anions in Cepheus and Auriga. Astrophysical Journal Letters, 730, [L18]. https://doi.org/10.1088/2041-8205/730/2/L18

\section{Published in:}

Astrophysical Journal Letters

\section{Document Version:}

Publisher's PDF, also known as Version of record

\section{Queen's University Belfast - Research Portal:}

Link to publication record in Queen's University Belfast Research Portal

\section{Publisher rights}

Copyright 2011 American Astronomical Society.

This work is made available online in accordance with the publisher's policies. Please refer to any applicable terms of use of the publisher.

\section{General rights}

Copyright for the publications made accessible via the Queen's University Belfast Research Portal is retained by the author(s) and / or other copyright owners and it is a condition of accessing these publications that users recognise and abide by the legal requirements associated with these rights.

Take down policy

The Research Portal is Queen's institutional repository that provides access to Queen's research output. Every effort has been made to ensure that content in the Research Portal does not infringe any person's rights, or applicable UK laws. If you discover content in the Research Portal that you believe breaches copyright or violates any law, please contact openaccess@qub.ac.uk. 


\title{
DISCOVERY OF INTERSTELLAR ANIONS IN CEPHEUS AND AURIGA
}

\author{
M. A. Cordiner ${ }^{1}$, S. B. Charnley ${ }^{1}$, J. V. BucKle ${ }^{2}$, C. Walsh ${ }^{3}$, and T. J. Millar ${ }^{3}$ \\ ${ }^{1}$ Astrochemistry Laboratory and The Goddard Center for Astrobiology, NASA Goddard Space Flight Center, Code 691, 8800 Greenbelt Road, \\ Greenbelt, MD 20771, USA; martin.cordiner@nasa.gov \\ ${ }^{2}$ Cavendish Astrophysics Group and Kavli Institute for Cosmology, Institute of Astronomy, University of Cambridge, Madingley Road, Cambridge, CB3 0HE, UK \\ ${ }^{3}$ Astrophysics Research Centre, School of Mathematics and Physics, Queen's University Belfast, Belfast, BT7 1NN, UK \\ Received 2010 September 27; accepted 2011 January 14; published 2011 March 4
}

\begin{abstract}
We report the detection of microwave emission lines from the hydrocarbon anion $\mathrm{C}_{6} \mathrm{H}^{-}$and its parent neutral $\mathrm{C}_{6} \mathrm{H}$ in the star-forming region L1251A (in Cepheus), and the pre-stellar core L1512 (in Auriga). The carbonchain-bearing species $\mathrm{C}_{4} \mathrm{H}, \mathrm{HC}_{3} \mathrm{~N}, \mathrm{HC}_{5} \mathrm{~N}, \mathrm{HC}_{7} \mathrm{~N}$, and $\mathrm{C}_{3} \mathrm{~S}$ are also detected in large abundances. The observations of L1251A constitute the first detections of anions and long-chain polyynes and cyanopolyynes (with more than five carbon atoms) in the Cepheus Flare star-forming region, and the first detection of anions in the vicinity of a protostar outside of the Taurus molecular cloud complex, indicating a possible wider importance for anions in the chemistry of star formation. Rotational excitation temperatures have been derived from the $\mathrm{HC}_{3} \mathrm{~N}$ hyperfine structure lines and are found to be $6.2 \mathrm{~K}$ for L1251A and $8.7 \mathrm{~K}$ for L1512. The anion-to-neutral ratios are $3.6 \%$ and $4.1 \%$, respectively, which are within the range of values previously observed in the interstellar medium, and suggest a relative uniformity in the processes governing anion abundances in different dense interstellar clouds. This research contributes toward the growing body of evidence that carbon chain anions are relatively abundant in interstellar clouds throughout the Galaxy, but especially in the regions of relatively high density and high depletion surrounding pre-stellar cores and young, embedded protostars.
\end{abstract}

Key words: astrochemistry - ISM: abundances - ISM: clouds - ISM: molecules - stars: formation

\section{INTRODUCTION}

Hydrocarbon anions have recently been discovered in the quiescent molecular clouds TMC-1 (McCarthy et al. 2006) and Lupus-1A (Sakai et al. 2010), the carbon-rich asymptotic giant branch star IRC+10216 (Cernicharo et al. 2007; Remijan et al. 2007), the protostars L1527 and L1521F, and the pre-stellar core L1544 (Sakai et al. 2008b; Gupta et al. 2009). The possibility that an appreciable fraction of molecular material in interstellar clouds might be in the form of anions was first suggested by Sarre (1980) and Herbst (1981), who pointed out that carbon chain molecules and other radicals have large electron affinities, leading to large radiative attachment rates such as those measured by Woodin et al. (1980). However, the full significance of anions for astrochemistry is still far from understood, so we seek to address the question of just how widespread anions are in the Galaxy. Apart from the single detection in Lupus (Sakai et al. 2010), all interstellar anion detections to date have been confined to the Taurus molecular cloud complex. The origin of the large observed abundances of polyynes $\left(\mathrm{C}_{n} \mathrm{H}, n=2-8\right)$ and cyanopolyynes $\left(\mathrm{HC}_{2 n+1} \mathrm{~N}, n=1-5\right)$ in this region (e.g., Cernicharo et al. 1986; Bell et al. 1998; Brünken et al. 2007) is debated, and may be attributable to a young gas-phase chemistry (Herbst \& Leung 1989), interactions between gas and dust (e.g., Brown \& Charnley 1991), or shocking in cloud-cloud collisions (Little et al. 1978). Walsh et al. (2009) theorized that the observed hydrocarbon anions act as catalysts for the synthesis of increased polyyne and cyanopolyyne abundances. New observations of long carbon chains and anions outside of the Taurus complex will provide tests for these astrochemical models and will provide data for the development of new theories regarding the formation of carbon chains throughout the Galaxy.

Models for anion chemistry are able to reproduce, with reasonable accuracy, the observed abundances of $\mathrm{C}_{6} \mathrm{H}^{-}$and $\mathrm{C}_{8} \mathrm{H}^{-}$ in TMC-1, IRC+10216, and L1527 (Millar et al. 2007; Remijan et al. 2007; Harada \& Herbst 2008; Cordiner et al. 2008). The recent detection of $\mathrm{CN}^{-}$in IRC+10216 by Agúndez et al. (2010) also matches well the abundance predicted by the chemical model of Cordiner \& Millar (2009). However, there are still discrepancies between the modeled and observed anion-to-neutral ratios, especially for $\mathrm{C}_{4} \mathrm{H}^{-}$(Herbst \& Osamura 2008), and the lack of anions in photondominated regions (Agúndez et al. 2008) is at variance with the model predictions of Millar et al. (2007). Clearly, our understanding of molecular anion chemistry is incomplete. Anions may be of wider importance in astrophysics; their presence within magnetized, collapsing cores may have consequences for star formation dynamics, through their influence on the ambipolar diffusion rate. Molecular anions, including $\mathrm{CH}_{2} \mathrm{CN}^{-}$, have been considered as plausible carriers for at least some of the unidentified diffuse interstellar bands (Cordiner \& Sarre 2007). Anion abundances are theorized to be sensitive to electron attachment and photodetachment rates (see Millar et al. 2007), and may therefore provide a useful tool for the determination of accurate electron densities and cosmic ray/ photoionization rates in astrophysical environments.

Presently, many of the key reactions and rates relevant to molecular anion chemistry, such as those of radiative electron attachment, have not been well studied in the laboratory, and so are poorly constrained or grossly approximated in chemical models. Given the current lack of laboratory experiments and detailed (quantum) theoretical calculations, the only way to constrain these parameters and further our understanding of the role of anions in astrophysics is by radio observations and complementary chemical modeling of molecular anions in various astrophysical environments.

This Letter reports new detections of the carbon chain anion $\mathrm{C}_{6} \mathrm{H}^{-}$in the vicinity of a young, low-mass protostar in the dense molecular cloud L1251A (in the Cepheus complex) and in the pre-stellar core L1512 (in the Taurus-Auriga complex). 


\section{OBSERVATIONS}

Sources were selected from a set of 16 dense clouds for which $\mathrm{HC}_{3} \mathrm{~N} J=10-9$ maps had previously been obtained using the Onsala $20 \mathrm{~m}$ telescope between 2005 and 2007 (some of which were published by Buckle et al. 2006). The close chemical relationship between polyynes and cyanopolyynes (see, e.g., Federman et al. 1990; Millar \& Herbst 1994) suggests that $\mathrm{C}_{4} \mathrm{H}$ and $\mathrm{C}_{6} \mathrm{H}$ should be abundant in dense molecular clouds, close to where the $\mathrm{HC}_{3} \mathrm{~N}$ peaks. Therefore, to improve the chances of successfully detecting $\mathrm{C}_{4} \mathrm{H}, \mathrm{C}_{6} \mathrm{H}$, and $\mathrm{C}_{6} \mathrm{H}^{-}$compared with previous surveys (e.g., Gupta et al. 2009), we targeted the strongest $\mathrm{HC}_{3} \mathrm{~N}$ emission peaks in these maps, which, due to chemical differentiation (e.g., Buckle et al. 2006; Bergin et al. 2007), are not generally coincident with the locations of peak emission from commonly observed molecules such as $\mathrm{NH}_{3}$, $\mathrm{N}_{2} \mathrm{H}^{+}$, or $\mathrm{CO}$ (for which maps already exist in the literature). Onsala $\mathrm{HC}_{3} \mathrm{~N} J=10-9$ maps of L1251A and L1512 are shown in Figure 1. The adopted coordinates for our anion and carbon chain searches were L1251A: R.A. $(2000)=22: 30: 40.4$, decl. $(2000)=+75: 13: 46$; L1512: R.A. $(2000)=5: 04: 07.1$, decl. $(2000)=32: 43: 09$. The L1251A position lies $40^{\prime \prime}$ from the center of the Class 0 protostar L1251A IRS3 and the L1512 position lies $26^{\prime \prime}$ from the center of a $\left(\approx 120^{\prime \prime}\right.$ wide) pre-stellar core (Di Francesco et al. 2008; Kirk et al. 2005). The positions observed by Gupta et al. (2009; who did not detect $\mathrm{C}_{6} \mathrm{H}^{-}$in either cloud), are not coincident with our observed positions.

Observations were carried out in the months of 2010 April and June using the NRAO $100 \mathrm{~m}$ Green Bank Telescope (GBT) ${ }^{4}$ The Ka receiver was used with $50 \mathrm{MHz}$ bandwidth and 8192 channels (corresponding to a channel spacing of $\approx 0.065 \mathrm{~km} \mathrm{~s}^{-1}$ ), in each of four spectral windows. In the middle of the observed frequency range $(28 \mathrm{GHz})$, the telescope beam FWHM was $26^{\prime \prime}$ and the beam efficiency factor was 0.88 . We performed deep integrations to search for emission lines from $\mathrm{C}_{4} \mathrm{H}, \mathrm{C}_{6} \mathrm{H}, \mathrm{C}_{6} \mathrm{H}^{-}$, and $\mathrm{HC}_{7} \mathrm{~N}$. Additional observations were obtained of $\mathrm{HC}_{5} \mathrm{~N}, \mathrm{C}_{3} \mathrm{~S}$, and $\mathrm{CH}_{3} \mathrm{CCH}$. For the compact, spatially isolated source L1512, beam switching (with 78" throw) was used, and for the more extended source L1215A, frequency switching was used. Pointing was checked every one to two hr and was typically accurate to within $5^{\prime \prime}$. Total system temperatures were in the range of 40-60 K.

New maps of $\mathrm{HC}_{3} \mathrm{~N} J=10-9$ emission in L1251A and $\mathrm{C}_{4} \mathrm{H}$ $N=9-8$ emission in L1512 were obtained using the Onsala $20 \mathrm{~m}$ telescope in 2010 May (shown in Figure 1). The basic observation and reduction techniques were presented by Buckle et al. (2006).

\section{RESULTS}

The $\mathrm{C}_{6} \mathrm{H}^{-}$and $\mathrm{C}_{6} \mathrm{H}$ spectra observed in L1251A and L1512 are shown in Figure 2. To improve the signal-to-noise ratio for L1251A, the $\mathrm{C}_{6} \mathrm{H}^{-} J=10-9$ and $J=11-10$ spectra have been averaged in velocity space.

The observed spectral lines were least-squares fitted using single Gaussians, the parameters for which are given in Table 1. The five hyperfine peaks of the $\mathrm{HC}_{3} \mathrm{~N} J=3-2$ transition are well resolved in our spectra, from which we derived gas rotational excitation temperatures $\left(T_{e x}\right)$ of $6.2 \pm 0.3 \mathrm{~K}$ for L1251A and $8.7 \pm 0.7 \mathrm{~K}$ for L1512 (using Equations (1) and (2) of Savage et al. 2002). These temperatures were used in

\footnotetext{
4 The National Radio Astronomy Observatory is a facility of the National Science Foundation operated under cooperative agreement by Associated Universities, Inc.
}
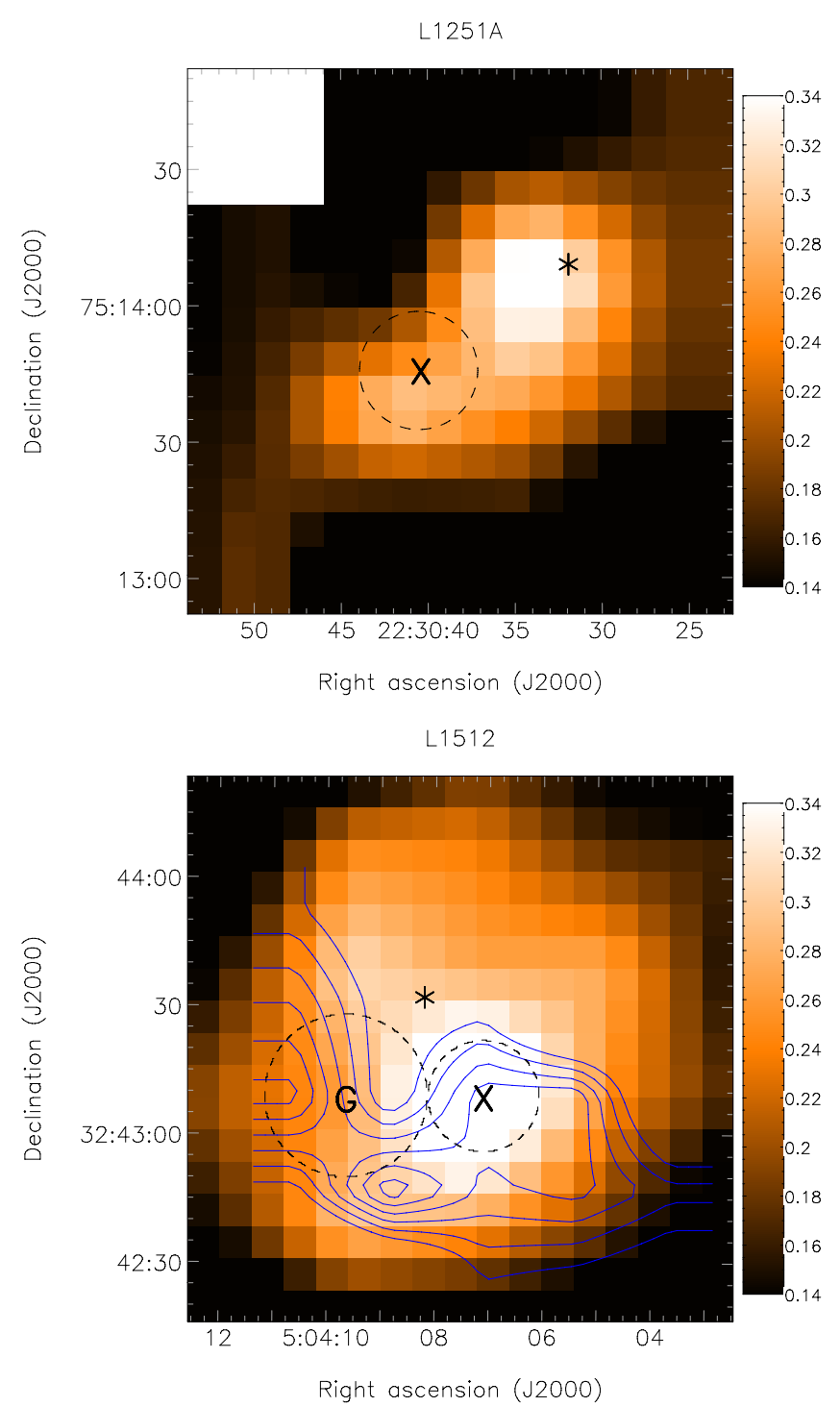

Figure 1. Onsala $\mathrm{HC}_{3} \mathrm{~N} J=10-9$ maps of L1251A (top) and L1512 with $\mathrm{C}_{4} \mathrm{H} \mathrm{N}=9-8$ contours overlaid (bottom). Scale bar units are $\mathrm{K} \mathrm{km} \mathrm{s}^{-1} \cdot \mathrm{C}_{4} \mathrm{H}$ contours show peak antenna temperature, starting at $0.36 \mathrm{~K}$, and increasing in steps of $0.02 \mathrm{~K}$. The observed GBT positions are labeled "X" and location of the Gupta et al. (2009) L1512 anion search is labeled " $G$ ". Asterisks show positions of protostar (for L1251A) and submillimeter source (for L1512). Dashed circles represent the GBT beam FWHM of the respective anion searches. Our chosen L1251A position does not coincide precisely with the $\mathrm{HC}_{3} \mathrm{~N}$ peak because it was based on an earlier, lower-sensitivity map than that shown.

the calculation of the column densities of the observed species assuming LTE and optically thin emission (see for example, Equation (2) of Lis et al. 2002). Where multiple transitions were observed for a given species, the average of the calculated column densities was taken, except for $\mathrm{HC}_{3} \mathrm{~N}$, for which only the optically thin $\Delta F=0$ transitions were used. The $\mathrm{HC}_{3} \mathrm{~N}$ and other recorded spectra will be presented in a future article. Column densities are given in Table 2.

\section{DISCUSSION}

Large abundances of carbon-chain-bearing species were observed in both L1251A and L1512. The measured $\mathrm{C}_{4} \mathrm{H}$ and $\mathrm{C}_{6} \mathrm{H}$ column densities (see Table 2) are similar to those in interstellar clouds with the highest known carbon chain abundances in the Galaxy (see Sakai et al. 2008a, 2009; Gupta et al. 2009). The abundance of carbon chains is also highlighted in L1512 
Table 1

Observed Line Parameters

\begin{tabular}{|c|c|c|c|c|c|c|c|c|c|}
\hline \multirow[t]{2}{*}{ Species Transition $^{a}$} & \multirow[t]{2}{*}{ Frequency } & \multicolumn{4}{|c|}{ L1512 } & \multicolumn{4}{|c|}{ L1251A } \\
\hline & & $T_{a}$ & $v$ & $\Delta v$ & $\int T_{a} d v$ & $T_{a}$ & $v$ & $\Delta v$ & $\int T_{a} d v$ \\
\hline $\mathrm{C}_{6} \mathrm{H}^{-} 10-9$ & 27537.13 & $46(7)$ & 7.09 & $0.13(2)$ & $6(1)$ & $27(7)$ & -3.96 & $0.21(6)$ & $6(2)$ \\
\hline $\mathrm{C}_{6} \mathrm{H}^{-} 11-10$ & 30290.81 & $18(7)$ & 7.10 & $0.16(7)$ & $3(1)$ & $24(7)$ & -4.00 & $0.18(5)$ & $5(1)$ \\
\hline $\mathrm{C}_{4} \mathrm{H} 3-2,3.5-2.5,3-2$ & 28532.31 & $715(50)$ & 7.05 & $0.15(1)$ & $114(8)$ & $383(45)$ & -4.05 & $0.38(5)$ & $155(18)$ \\
\hline $\mathrm{C}_{4} \mathrm{H} 3-2,3.5-2.5,4-3$ & 28532.46 & $931(50)$ & 7.06 & $0.15(1)$ & $149(8)$ & $486(34)$ & -4.04 & $0.41(3)$ & $212(15)$ \\
\hline $\mathrm{C}_{6} \mathrm{H} 10.5-9.5, f, 11-10$ & 29109.64 & $91(7)$ & 7.26 & $0.13(1)$ & $13(1)$ & $56(6)$ & -3.85 & $0.32(4)$ & $19(2)$ \\
\hline $\mathrm{C}_{6} \mathrm{H} 10.5-9.5, f, 10-9$ & 29109.69 & $84(6)$ & 7.01 & $0.16(1)$ & $14(1)$ & $58(6)$ & -4.11 & $0.27(3)$ & $17(2)$ \\
\hline $\mathrm{C}_{6} \mathrm{H} 10.5-9.5, e, 11-10$ & 29112.71 & $99(7)$ & 7.19 & $0.14(1)$ & $15(1)$ & $58(6)$ & -3.94 & $0.28(3)$ & $17(2)$ \\
\hline $\mathrm{C}_{6} \mathrm{H} 10.5-9.5, e, 10-9$ & 29112.75 & $84(7)$ & 6.96 & $0.15(1)$ & $13(1)$ & $52(6)$ & -4.15 & $0.33(4)$ & $18(2)$ \\
\hline $\mathrm{HC}_{3} \mathrm{~N} 3-2,3-3$ & 27292.90 & $671(65)$ & 7.07 & $0.12(1)$ & $86(11)$ & $246(24)$ & -3.99 & $0.31(3)$ & $81(11)$ \\
\hline $\mathrm{HC}_{3} \mathrm{~N} 3-2,2-1$ & 27294.07 & $2102(52)$ & 6.98 & $0.16(0)$ & $358(9)$ & $967(22)$ & -4.10 & $0.37(1)$ & $382(10)$ \\
\hline $\mathrm{HC}_{3} \mathrm{~N} 3-2,3-2$ & 27294.29 & $2769(53)$ & 7.07 & $0.16(0)$ & $472(9)$ & $1331(23)$ & -4.00 & $0.34(1)$ & $483(9)$ \\
\hline $\mathrm{HC}_{3} \mathrm{~N} 3-2,4-3$ & 27294.35 & $3607(54)$ & 7.15 & $0.16(0)$ & $614(9)$ & $1760(22)$ & -3.93 & $0.39(1)$ & $732(10)$ \\
\hline $\mathrm{HC}_{3} \mathrm{~N} 3-2,2-2$ & 27296.23 & $525(53)$ & 7.08 & $0.16(2)$ & 89 (14) & $246(66)$ & -4.02 & $0.36(11)$ & $94(38)$ \\
\hline $\mathrm{HC}_{5} \mathrm{~N} 11-10$ & 29289.15 & $1364(40)$ & 7.05 & $0.21(1)$ & $305(8)$ & $998(22)$ & -4.05 & $0.37(1)$ & $393(9)$ \\
\hline $\mathrm{HC}_{5} \mathrm{~N} 12-11$ & 31951.77 & $1650(23)$ & 7.08 & $0.20(0)$ & $351(5)$ & $\ldots$ & $\ldots$ & $\ldots$ & $\ldots$ \\
\hline $\mathrm{HC}_{5} \mathrm{~N} 13-12$ & 34614.39 & $1662(24)$ & 7.13 & $0.18(0)$ & $318(5)$ & $\ldots$ & $\ldots$ & $\ldots$ & $\ldots$ \\
\hline $\mathrm{HC}_{7} \mathrm{~N} 25-24$ & 28199.81 & $202(8)$ & 7.20 & $0.14(1)$ & $30(1)$ & $123(11)$ & -3.92 & $0.29(3)$ & $38(3)$ \\
\hline $\mathrm{C}_{3} \mathrm{~S} 5-4$ & 28903.69 & $573(45)$ & 7.16 & $0.15(1)$ & $92(7)$ & $517(21)$ & -4.01 & $0.33(2)$ & $182(7)$ \\
\hline $\mathrm{CH}_{3} \mathrm{CCH} 2_{1}-1_{1}$ & 34182.76 & $252(25)$ & 7.13 & $0.19(2)$ & $51(5)$ & $\ldots$ & $\ldots$ & $\ldots$ & $\ldots$ \\
\hline $\mathrm{CH}_{3} \mathrm{CCH} 2_{0}-1_{0}$ & 34183.42 & $264(22)$ & 7.15 & $0.23(2)$ & $65(5)$ & $\ldots$ & $\ldots$ & $\ldots$ & $\ldots$ \\
\hline
\end{tabular}

Notes. Units of $T_{a}$ are in $\mathrm{mK}$ and velocities are in $\mathrm{km} \mathrm{s}^{-1}$, relative to the LSR frame. The $1 \sigma$ errors on the last quoted digit(s) are given in parentheses. a Transitions are specified as follows: $\mathrm{C}_{6} \mathrm{H}^{-}, \mathrm{HC}_{5} \mathrm{~N}, \mathrm{HC}_{7} \mathrm{~N}$, and $\mathrm{C}_{3} \mathrm{~S}: J^{\prime \prime}-J^{\prime} ; \mathrm{C}_{6} \mathrm{H}: J^{\prime \prime}-J^{\prime}$, parity, $F^{\prime \prime}-F^{\prime} ; \mathrm{C}_{4} \mathrm{H}: N^{\prime \prime}-N^{\prime}, J^{\prime \prime}-J^{\prime}, F^{\prime \prime}-F^{\prime} ; \mathrm{HC}_{3} \mathrm{~N}$ : $J^{\prime \prime}-J^{\prime}, F^{\prime \prime}-F^{\prime} ; \mathrm{CH}_{3} \mathrm{CCH}: J_{K}^{\prime \prime}-J_{K}^{\prime}$.
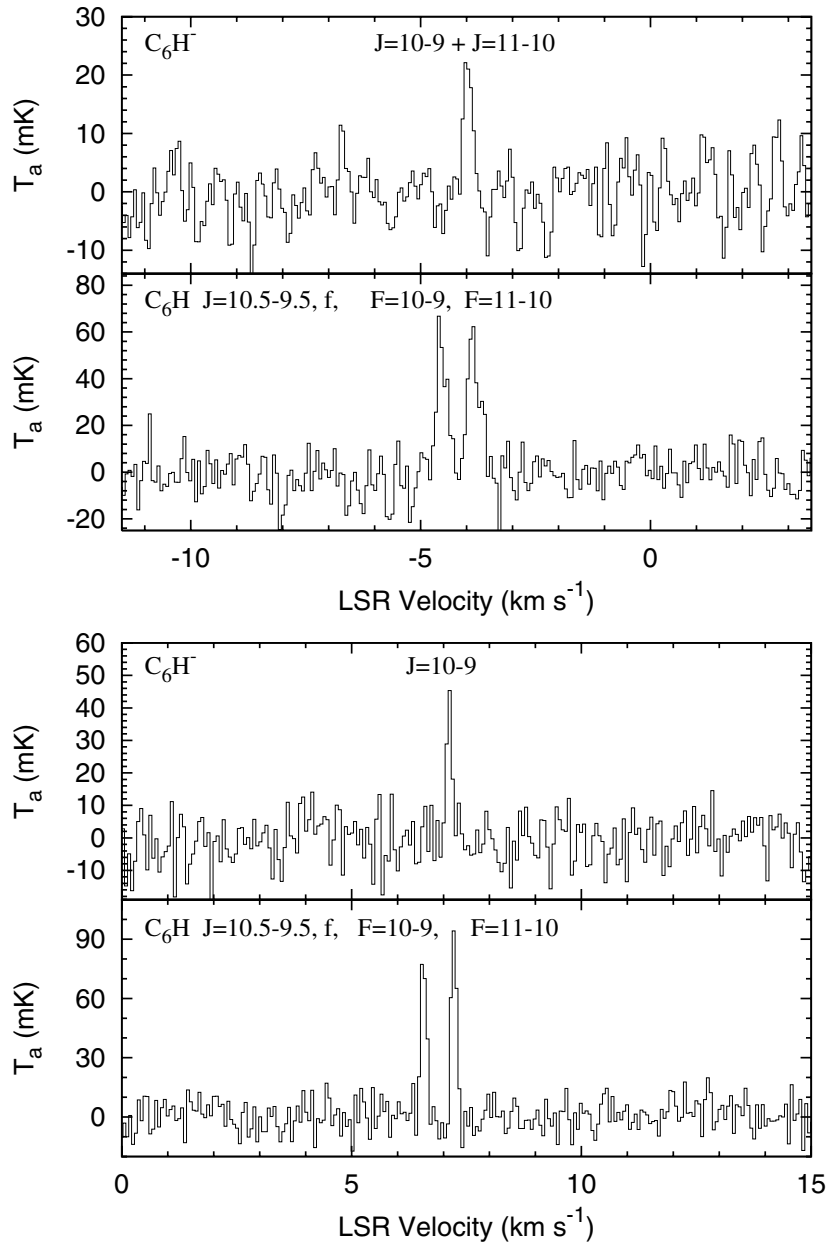

Figure 2. Plot showing (averaged) $\mathrm{C}_{6} \mathrm{H}^{-} J=10-9$ and $J=11-10$ spectrum of L1251A (top) and $J=10-9$ spectrum of L1512 (bottom). The $\mathrm{C}_{6} \mathrm{H}$ $J=10.5-9.5, f$ spectra are also shown.
Table 2

Observed Molecular Column Densities

\begin{tabular}{lcc}
\hline \hline Species & L1512 & L1251A \\
\hline $\mathrm{C}_{6} \mathrm{H}^{-}$ & $2.0 \pm 0.5 \times 10^{10}$ & $2.7 \pm 0.7 \times 10^{10}$ \\
$\mathrm{C}_{6} \mathrm{H}$ & $4.8 \pm 0.3 \times 10^{11}$ & $7.6 \pm 0.8 \times 10^{11}$ \\
$\mathrm{C}_{4} \mathrm{H}$ & $9.2 \pm 0.6 \times 10^{13}$ & $1.2 \pm 0.1 \times 10^{14}$ \\
$\mathrm{HC}_{3} \mathrm{~N}$ & $3.0 \pm 0.4 \times 10^{13}$ & $2.8 \pm 0.8 \times 10^{13}$ \\
$\mathrm{HC}_{5} \mathrm{~N}$ & $4.9 \pm 0.1 \times 10^{12}$ & $7.5 \pm 0.2 \times 10^{12}$ \\
$\mathrm{HC}_{7} \mathrm{~N}$ & $1.9 \pm 0.1 \times 10^{12}$ & $4.7 \pm 0.4 \times 10^{12}$ \\
$\mathrm{C}_{3} \mathrm{~S}$ & $1.3 \pm 0.1 \times 10^{12}$ & $2.6 \pm 0.1 \times 10^{12}$ \\
$\mathrm{CH}_{3} \mathrm{CCH}$ & $3.1 \pm 0.3 \times 10^{13}$ & $\ldots$
\end{tabular}

Note. Units are $\mathrm{cm}^{-2}$.

by the large $\mathrm{CH}_{3} \mathrm{CCH}$ column density, which is comparable to that found in L1527 and TMC-1 (Sakai et al. 2008a). The $\mathrm{C}_{3} \mathrm{~S}$ column densities in both L1251A and L1512 are somewhat less than previously observed in "carbon-chain-producing regions" by Hirota \& Yamamoto (2006). Possible explanations for this include depletion onto dust or reduced elemental sulphur abundances.

The derived $\mathrm{C}_{6} \mathrm{H}^{-}$column densities of $(2.7 \pm 0.7) \times$ $10^{10} \mathrm{~cm}^{-2}$ in L1251A and $(2.0 \pm 0.5) \times 10^{10} \mathrm{~cm}^{-2}$ in L1512 are similar to those previously observed in other low-mass starforming cores, e.g., L1521F and L1544 $\left(3.4 \times 10^{10} \mathrm{~cm}^{-2}\right.$ and $3.1 \times 10^{10} \mathrm{~cm}^{-2}$, respectively; Gupta et al. 2009), and L1527 $\left(5.8 \times 10^{10} \mathrm{~cm}^{-2}\right.$; Sakai et al. 2007). These values are somewhat less than those observed in the quiescent molecular clouds TMC-1 $\left(1.2 \times 10^{11} \mathrm{~cm}^{-2}\right.$; Brünken et al. 2007) and Lupus-1A $\left(6.5 \times 10^{10} \mathrm{~cm}^{-2}\right.$; Sakai et al. 2010). The anion-to-neutral ratios $\left(\left[\mathrm{C}_{6} \mathrm{H}^{-}\right] /\left[\mathrm{C}_{6} \mathrm{H}\right]\right)$, however, are approximately in the middle of the previously observed distribution $3.6 \% \pm 1.3 \%$ for L1251A and $4.2 \% \pm 1.4 \%$ for L1512, compared with $1.6 \%$ for TMC-1, 2.1\% for Lupus-1A, 2.5\% for L1544, 4\% for L1521F, and $9.3 \%$ for L1527. Sakai et al. $(2007,2010)$ hypothesized that an inverse relationship between $\mathrm{H}$-atom abundance and gas density would result in greater $\left[\mathrm{C}_{6} \mathrm{H}^{-}\right] /\left[\mathrm{C}_{6} \mathrm{H}\right]$ ratios in 
denser gas, which may explain the larger ratio found in L1527 where the hydrogen nucleon density $n_{H}$ is $\sim 10^{6} \mathrm{~cm}^{-3}$, compared to $n_{H} \sim 10^{4} \mathrm{~cm}^{-3}$ in TMC-1. L1251A and L1512 fit this trend-both have $n_{H} \sim 10^{5} \mathrm{~cm}^{-3}$ (Lee et al. 2010; Kirk et al. 2005), and their anion-to-neutral ratios are intermediate between TMC-1 and L1527.

The detection of $\mathrm{C}_{6} \mathrm{H}^{-}$in $\mathrm{L} 1251 \mathrm{~A}$ is the first reported interstellar anion in a protostar outside of Taurus, and thus shows that the importance of anions must be considered in future studies of the chemistry of star-forming regions throughout the Galaxy.

L1251A and L1512 have similar $\left[\mathrm{C}_{6} \mathrm{H}\right] /\left[\mathrm{C}_{4} \mathrm{H}\right]$ ratios of $0.6 \%$ and $0.5 \%$, respectively. These are within the range of values previously measured in interstellar clouds by Gupta et al. (2009).

\section{1. $L 1251 A$}

The L1251A molecular cloud is located in the Cepheus Flare region of low-to-intermediate mass star formation (Kun et al. 2008). Our observed position is $40^{\prime \prime} \mathrm{SE}$ of the embedded Class 0 protostar L1251A IRS3, which powers a molecular outflow (Lee et al. 2010). The protostar center is located outside of the 26" GBT beam (see Figure 1), so emission from its core does not directly influence our observations. However, depending on the radius of its outer boundary (which is likely to be up to a few times $10^{4}$ AU; see, e.g., Jørgensen et al. 2002), gas from inside the protostar envelope is probably responsible for much of the observed molecular emission. It would be of interest to observe the carbon chain (and anion) emission closer to the protostellar core, to determine whether the elevated temperatures there have any impact on the abundances of these species, as has been hypothesized by Sakai et al. (2008a).

Although previous observations have shown relatively large $\mathrm{HC}_{3} \mathrm{~N}$ and $\mathrm{HC}_{5} \mathrm{~N}$ abundances in various parts of the Cepheus complex, our observations constitute the largest reported $\mathrm{C}_{4} \mathrm{H}$ column density and the first detection of the long-chain species $\mathrm{C}_{6} \mathrm{H}$ and $\mathrm{HC}_{7} \mathrm{~N}$ in this region. The derived $\left[\mathrm{HC}_{7} \mathrm{~N}\right] /\left[\mathrm{HC}_{5} \mathrm{~N}\right]$ ratio of $63 \%$ is exceptionally high compared with other dense interstellar clouds: the largest value observed in the seven carbon-chain-rich clouds reported by Hirota \& Yamamoto (2006) and Sakai et al. (2008a) is 37\% in TMC-1. However, there is some uncertainty in the excitation temperatures of these species, as they have been found to differ from each other in TMC-1 by about 1-3 K (e.g., Bell et al. 1998). Assuming that the $\mathrm{HC}_{5} \mathrm{~N}$ rotational temperature is $4-5 \mathrm{~K}$ and the $\mathrm{HC}_{7} \mathrm{~N}$ temperature is 5-7 K, and accounting for the optical depth of the $\mathrm{HC}_{5} \mathrm{~N}$ line $(0.3)$, the resulting $\left[\mathrm{HC}_{7} \mathrm{~N}\right] /\left[\mathrm{HC}_{5} \mathrm{~N}\right]$ ratio in L1251A is $17 \%-89 \%$. Cernicharo et al. (1986) observed an average ratio of $28 \%$ in six cloudlets in Taurus. Thus, the ratio in L1251A is likely to be similar to or larger than in Taurus. These results show that chemical conditions in the Cepheus Flare can be highly conducive to the formation of relatively long carbonchain-bearing species.

The profiles of the spectral lines in L1251A show a small amount of asymmetry-insufficient to justify fitting multiple Gaussians, but nevertheless indicative of a more complex cloud structure. Although we derive a lower temperature for this cloud than L1512, the lines are broader by approximately a factor of two, which indicates the presence of significant bulk motions of the gases along the line of sight. This may be related to turbulence arising from the L1251A IRS3 outflow, which partially intersects our observed telescope beam.

There is some evidence that the $\mathrm{C}_{6} \mathrm{H}^{-}$lines (with $\Delta v \approx$ $0.20 \mathrm{~km} \mathrm{~s}^{-1}$ ) are narrower in this source than the lines of the neutral species (with $\Delta v \approx 0.30 \mathrm{~km} \mathrm{~s}^{-1}$ ). A similar phenomenon was also observed by Sakai et al. (2010) in Lupus-1A, who suggested that this may be due to the anion being preferentially located in cooler, denser gas than the neutral. This suggestion seems reasonable on the basis that the rate of radiative electron attachment and, thus, the rate of anion formation is theorized to be proportional to $T^{-1 / 2}$ and to the electron density (Herbst \& Osamura 2008).

\section{2. $L 1512$}

L1512 is a rather isolated molecular cloud core located in the nearby Taurus-Auriga complex (Ungerechts \& Thaddeus 1987). It contains a compact (FWHM $\left.\sim 10^{4} \mathrm{AU}\right)$ submillimeter source centered around R.A. (2000) = 5:04:08.2, decl. (2000) $=$ +32:43:32 (Di Francesco et al. 2008; Kirk et al. 2005), 26" NE of our observed position (see Figure 1), which indicates the presence of a dense pre-stellar core covering our telescope beam. Gupta et al. (2009) previously attempted to detect $\mathrm{C}_{6} \mathrm{H}^{-}$at the Benson \& Myers (1989) position (30" east of our observed position, as shown in Figure 1), and they derived an upper limit of $4.8 \times 10^{10} \mathrm{~cm}^{-2}$, which is consistent with our observed column density (see Table 2). They also derived $\mathrm{C}_{4} \mathrm{H}$ and $\mathrm{C}_{6} \mathrm{H}$ column densities of $(9 \pm 3) \times 10^{13} \mathrm{~cm}^{-2}$ and $(5 \pm 2) \times 10^{11} \mathrm{~cm}^{-2}$, respectively, which closely match our observed values. Hirota et al. (2009) measured an $\mathrm{HC}_{3} \mathrm{~N}$ column density of $2.5 \times 10^{13} \mathrm{~cm}^{-2}$, $10^{\prime \prime}$ west of our L1512 position, which is in reasonably good agreement with our observed value. The similarities of our observed column densities with those found nearby indicate that polyyne and cyanopolyyne abundances may be less variable than shown by the $\mathrm{HC}_{3} \mathrm{~N}$ map in Figure 1, over spatial scales of $\sim 30^{\prime \prime}$ (which corresponds to $0.02 \mathrm{pc}$ at the distance of L1512). This implies that the strong, compact peak shown in the $\mathrm{HC}_{3} \mathrm{~N}$ map may arise at least partly as a result of enhanced gas densities and/or temperatures in that region, which (in the case of subcritical line excitation) would cause increased $\mathrm{HC}_{3} \mathrm{~N} J=10-9$ emission as a result of increased excitation of the relatively high energy $(24 \mathrm{~K}) J=10$ rotational level.

\subsection{Comparison and Carbon Chemistry}

Located in two distinctly separate parts of the sky (L1251A is $\sim 330$ pc distant in Cepheus, whereas L1512 is $\sim 140$ pc distant in Auriga), it is surprising how chemically similar these two clouds are. Apart from $\mathrm{HC}_{7} \mathrm{~N}$, all of the observed column densities are within a factor of two of each other (see Table 2). This also applies to $\mathrm{NH}_{3}$ (Benson \& Myers 1989) and $\mathrm{N}_{2} \mathrm{H}^{+}$(Caselli et al. 2002). The large anion, polyyne, and cyanopolyyne abundances are indicative of an active carbon chemistry, comparable to that observed in other carbon-chainrich regions of the Galaxy (see, e.g., Hirota \& Yamamoto 2006 and Hirota et al. 2009).

Large abundances of unsaturated carbon chains are theorized to occur either in the relatively early stages of dark cloud evolution when carbon is abundant in reactive form (see, for example, Herbst \& Leung 1989) or later on during the "freezeout peak" (Brown \& Charnley 1990; Ruffle et al. 1997). In the latter scenario, the lighter, more reactive elements such as atomic oxygen are theorized to freeze out onto the dust grains over time. The loss of oxygen from the gas-phase results in reduced destruction rates for the carbon-chain-bearing species and their associated reagents, giving rise to elevated abundances of these species later on in a dense cloud's evolution.

Over time, two of the primary destructive reactants for organic anions-atomic hydrogen and oxygen-are lost from the gas 
phase through conversion on dust grain surfaces to $\mathrm{H}_{2}$ and $\mathrm{H}_{2} \mathrm{O}$, respectively (Goldsmith \& Li 2005; Bergin et al. 2000). This may explain why the $\mathrm{C}_{6} \mathrm{H}^{-}$anion-to-neutral ratios are greater in denser clouds (including L1521A, L1512, and L1527), than in the quiescent, lower-density clouds TMC-1 and Lupus-1A. Oxygen and hydrogen react quickly with $\mathrm{C}_{6} \mathrm{H}^{-}$(Eichelberger et al. 2007), so the combined effects of the depletion of $\mathrm{O}$ and $\mathrm{H}$ in these relatively more dense environments may be responsible for the large observed anion-to-neutral ratios in protostars and pre-stellar cores.

Alternatively, elevated carbon chain abundances could arise as a consequence of selective methane sublimation from dust grain surfaces, as originally investigated by Millar \& Freeman (1984) and Brown \& Charnley (1991). It is hypothesized that as a protostellar object contracts and begins to heat the surrounding gas, sublimated methane reacts with $\mathrm{C}^{+}$to form hydrocarbon ions, which gives rise to a so-called warm carbon chain chemistry (WCCC) via subsequent ion-molecule reactions (see Sakai et al. 2008a; Hassel et al. 2008). Given the close proximity of our observed L1251A position to the protostar IRS3, the WCCC theory may be applicable as a possible explanation for the large hydrocarbon abundances present there.

The location of our L1512 anion detection coincides with the region of CO depletion identified by Buckle et al. (2006). This is also the case for the anion and carbon chain detections in L1521F and L1544 made by Gupta et al. (2009). Thus, the "freeze-out peak" chemistry may be the preferred explanation for the large anion and carbon chain abundances observed in L1512 and similar pre-stellar cores. The presence of hydrocarbon anions is likely to be partly responsible for the large (neutral) polyyne and cyanopolyyne abundances in L1251A and L1512 (see Walsh et al. 2009).

\section{CONCLUSION}

We have detected the carbon chain anion $\mathrm{C}_{6} \mathrm{H}^{-}$for the first time in L1251A and L1512. Large column densities of the neutral species $\mathrm{C}_{4} \mathrm{H}, \mathrm{C}_{6} \mathrm{H}, \mathrm{HC}_{5} \mathrm{~N}, \mathrm{HC}_{7} \mathrm{~N}$, and $\mathrm{C}_{3} \mathrm{~S}$ were also detected. Combined with the five previous interstellar anion detections by McCarthy et al. (2006), Gupta et al. (2009), and Sakai et al. $(2007,2010)$, these results show that anions tend to be present in detectable quantities in regions where carbon chains are highly abundant, especially surrounding embedded protostars and pre-stellar cores. The anion-to-neutral ratios $\left[\mathrm{C}_{6} \mathrm{H}^{-}\right] /\left[\mathrm{C}_{6} \mathrm{H}\right]$ are consistently on the order of a few percent and show an apparent positive correlation with density. This implies that a relatively uniform and simple physical/ chemical mechanism is responsible for regulating molecular anion abundances in the different interstellar clouds observed so far.

The large polyyne and cyanopolyyne abundances measured in L1251A show that carbon-chain-production processes are rapid in this part of the Cepheus Flare molecular cloud complex, resulting in similar abundances to those found in the Taurus molecular cloud. The observation of abundant $\mathrm{C}_{6} \mathrm{H}^{-}$in L1251A constitutes the first detection of anions in a protostellar envelope outside of the Taurus-Auriga complex and indicates that anions are likely to be widespread throughout Galactic star-forming regions where carbon chains are present.

The technique of using $\mathrm{HC}_{3} \mathrm{~N}$ and $\mathrm{C}_{4} \mathrm{H}$ as proxies for the detection of less abundant, larger carbon chains and anions shows strong promise as a means for obtaining further detections of these molecules in low-mass star-forming regions in the future.
This research was supported by the NASA Exobiology Program and the Goddard Center for Astrobiology. Astrophysics at QUB is supported by a grant from STFC.

\section{REFERENCES}

Agúndez, M., Fonfría, J. P., Cernicharo, J., Pardo, J. R., \& Guélin, M. 2008, A\&A, 479, 493

Agúndez, M., et al. 2010, A\&A, 517, L2

Bell, M. B., Watson, J. K. G., Feldman, P. A., \& Travers, M. J. 1998, ApJ, 508, 286

Benson, P. J., \& Myers, P. C. 1989, ApJS, 71, 89

Bergin, E. A., \& Tafalla, M. 2007, ARA\&A, 45, 339

Bergin, E. A., et al. 2000, ApJ, 539, 129

Brown, P. D., \& Charnley, S. B. 1990, MNRAS, 244, 432

Brown, P. D., \& Charnley, S. B. 1991, MNRAS, 249, 69

Brünken, S., Gupta, H., Gottlieb, C. A., McCarthy, M. C., \& Thaddeus, P. 2007, ApJ, 664, L43

Buckle, J. V., Rodgers, S. D., Wirstrom, E. S., Charnley, S. B., MarkwickKemper, A. J., Butner, H. M., \& Takakuwa, S. 2006, Faraday Discuss., 133, 63

Caselli, P., Benson, P. J., Myers, P. C., \& Tafalla, M. 2002, ApJ, 572, 238

Cernicharo, J., Bachiller, R., \& Duvert, G. 1986, A\&A, 160, 181

Cernicharo, J., Guélin, M., Agúndez, M., Kawaguchi, K., McCarthy, M., \& Thaddeus, P. 2007, A\&A, 467, 37

Cordiner, M. A., \& Millar, T. J. 2009, ApJ, 697, 68

Cordiner, M. A., Millar, T. J., Walsh, C., Herbst, E., Lis, D. C., Bell, T. A., \& Roueff, E. 2008, in IAU Symp. 251, Organic Matter in Space, ed. S. Kwok \& S. Sandford (Cambridge: Cambridge Univ. Press), 157

Cordiner, M. A., \& Sarre, P. J. 2007, A\&A, 472, 537

Di Francesco, J., Johnstone, D., Kirk, H., MacKenzie, T., \& Ledwosinska, E. 2008, ApJS, 175, 227

Eichelberger, B., Snow, T. P., Barckholtz, C., \& Bierbaum, V. M. 2007, ApJ, 667,1283

Federman, S. R., Huntress, W. T., Jr., \& Prasad, S. S. 1990, ApJ, 354, 504

Goldsmith, P. F., \& Li, D. 2005, ApJ, 622, 938

Gupta, H., Gottlieb, C. A., McCarthy, M. C., \& Thaddeus, P. 2009, ApJ, 691, 1494

Harada, N., \& Herbst, E. 2008, ApJ, 685, 272

Hassel, G. E., Herbst, E., \& Garrod, R. T. 2008, ApJ, 681, 1385

Herbst, E. 1981, Nature, 289, 656

Herbst, E., \& Leung, C. M. 1989, ApJS, 69, 271

Herbst, E., \& Osamura, Y. 2008, ApJ, 679, 1670

Hirota, T., Ohishi, M., \& Yamamoto, S. 2009, ApJ, 699, 585

Hirota, T., \& Yamamoto, S. 2006, ApJ, 646, 258

Jørgensen, J. K., Schöier, F. L., \& van Dishoeck, E. F. 2002, A\&A, 389, 908

Kirk, J. M., Ward-Thompson, D., \& André, P. 2005, MNRAS, 360, 1506

Kun, M., Kiss, Z. T., \& Balog, Z. 2008, in Handbook of Star Forming Regions, Vol. I, ed. B. Reipurth (The Northern Sky ASP Monograph Publications, Vol. 4; San Francisco, CA: ASP), 136

Lee, J., et al. 2010, ApJ, 709, 74

Lis, D. C., Roueff, E., Gerin, M., Phillips, T. G., Coudert, L. H., van der Tak, F. F. S., \& Schilke, P. 2002, ApJ, 571, L55

Little, L. T., Riley, P. W., MacDonald, G. H., \& Matheson, D. N. 1978, MNRAS, 183,805

McCarthy, M. C., Gottlieb, C. A., Gupta, H. C., \& Thaddeus, P. 2006, ApJ, 652, L141

Millar, T. J., \& Freeman, A. 1984, MNRAS, 207, 405

Millar, T. J., \& Herbst, E. 1994, A\&A, 288, 561

Millar, T. J., Walsh, C., Cordiner, M. A., Ní Chuimín, R., \& Herbst, E. 2007, ApJ, 662, L87

Remijan, A. J., Hollis, J. M., Lovas, F. J., Cordiner, M. A., Millar, T. J., Markwick-Kemper, A. J., \& Jewell, P. R. 2007, ApJ, 664, L47

Ruffle, D. P., Hartquist, T. W., Taylor, S. D., \& Williams, D. A. 1997, MNRAS, 291,235

Sakai, N., Sakai, T., Hirota, T., \& Yamamoto, S. 2008a, ApJ, 672, 371

Sakai, N., Sakai, T., Hirota, T., \& Yamamoto, S. 2009, ApJ, 702, 1025

Sakai, N., Sakai, T., Osamura, Y., \& Yamamoto, S. 2007, ApJ, 667, L71

Sakai, N., Sakai, T., \& Yamamoto, S. 2008b, ApJ, 673, L71

Sakai, N., Shiino, T., Hirota, T., Sakai, T., \& Yamamoto, S. 2010, ApJ, 718, L49

Sarre, P. J. 1980, J. Chim. Phys., 77, 769

Savage, C., Apponi, A. J., Ziurys, L. M., \& Wyckoff, S. 2002, ApJ, 578, 211

Ungerechts, H., \& Thaddeus, P. 1987, ApJS, 63, 645

Walsh, C., Harada, N., Herbst, E., \& Millar, T. J. 2009, ApJ, 700, 725

Woodin, R., Foster, M. S., \& Beauchamp, J. L. 1980, J. Chem. Phys., 72, 4223 